and $w$-LSII are extremely small. These deviations seem to be caused by the lesser wettability of the diaphragm with the absorbent. Also, from the fact that the reaction product is crystallized only on Teflon diaphragm, it is suggested that the less wettable diaphragm suppresses the diffusion rate of the reaction product in diaphragm. Accordingly, it is necessary to note that only when the diaphragm used is very wettable with the absorbent a diaphragm cell is applicable to the study of gas absorption.

\section{Conclusion}

The absorption rate of $\mathrm{CO}_{2}$ into aqueous $\mathrm{NaOH}$ solution was measured by a diaphragm cell and compared with the results for transfer cells. It was found that the effect of the diaphragm itself inserted into the interface on the process of gas absorption is more pronounced for a diaphragm less wettable with the absorbent.

Nylon and Teflon diaphragms are not useful for aqueous solution as an absorbent, for example, but cellulose ester and polyvinyl chloride diaphragms are still useful. This implies that a diaphragm cell is applicable to the study of gas absorption, provided that the diaphragm used is very wettable with the absorbent.

Nomenclature

$\begin{array}{ll}a & =\text { geometric interfacial area } \\ B_{0} & =\text { initial concentration of } \mathrm{NaOH} \\ N_{A} & =\text { transient absorption rate }\end{array}$
$\left[\mathrm{mol} / \mathrm{cm}^{2} \cdot \mathrm{sec}\right]$

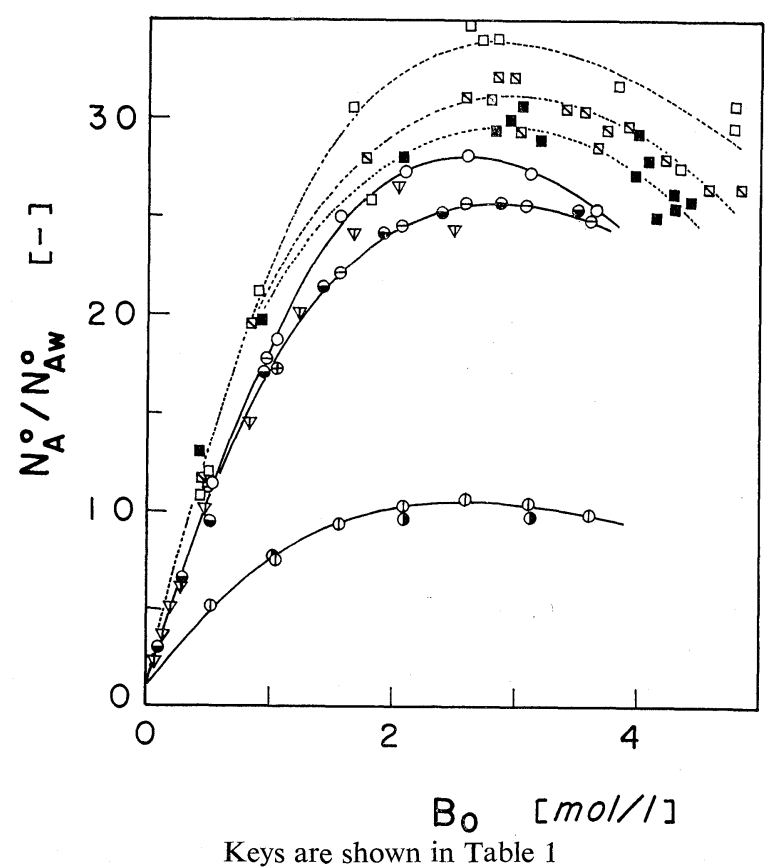

Fig. $1 N_{A}^{\circ} / N_{A}^{\circ} w$ vs. $B_{0}$ for $\mathrm{CO}_{2}-\mathrm{NaOH}$ system

$N_{A}^{\circ}, N_{A w}^{\circ}=$ extrapolated initial absorption rate into aqueous solution and water $\quad\left[\mathrm{mol} / \mathrm{cm}^{2} \cdot \mathrm{sec}\right]$

$\begin{array}{lll}t & =\text { gas-liquid contact time }\end{array}$

\section{Literature Cited}

1) Onda, K., H. Takeuchi and M. Fujine: J. Chem. Eng. Japan, 8, 25 (1975).

2) Sano, M.: Ph.D. Dissertation, Waseda Univ. (1969).

3) Vassilatos, G., O. Trass and A. I. Johnson: Can. J. Chem. Eng., 40, 210 (1962).

4) Whitman, W. G.: Chem. Met. Eng., 29, 166 (1923).

\title{
PARTICLE-TO-LIQUID MASS TRANSFER IN A STIRRED TANK WITH A BASKET IMPELLER
}

MotoyuKi SUZUKI* AND KUNITARo KAWAZOE Institute of Industrial Science, University of Tokyo, Tokyo, 106

The rate study of adsorption in an aqueous system is often carried out in a stirred tank contactor where adsorbent particles are suspended. When the intraparticle diffusion kinetic is of concern, one of the disadvantages of this system is the difficulty in fulfilling the condition of negligible mass transfer resistance between liquid and the surface of the particles $^{3)}$. Since suspended particles in the tank travel with the surrounding liquid and thus the relative velocity between the particle and the surrounding liquid cannot be large enough, there practically

\footnotetext{
Received June 4, 1974.
}

exists an upper limit of mass transfer coefficient between particle surface and bulk fluid. Furthermore, too vigorous agitation leads to cavitation in the liquid or particles size reduction due to attrition.

The present note suggests that a large mass transfer rate between particle surface and bulk fluid can be attained by using a basket impeller in which the particles are held. Contactors of this type have become common in catalyst studies in gaseous systems ${ }^{1,7)}$, but apparently no work has been done in liquid, where mass transfer resistance can be more significant. 


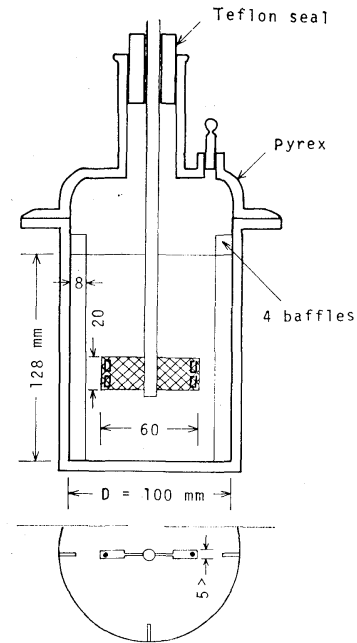

Fig. 1 Schematic illustration of the reactor

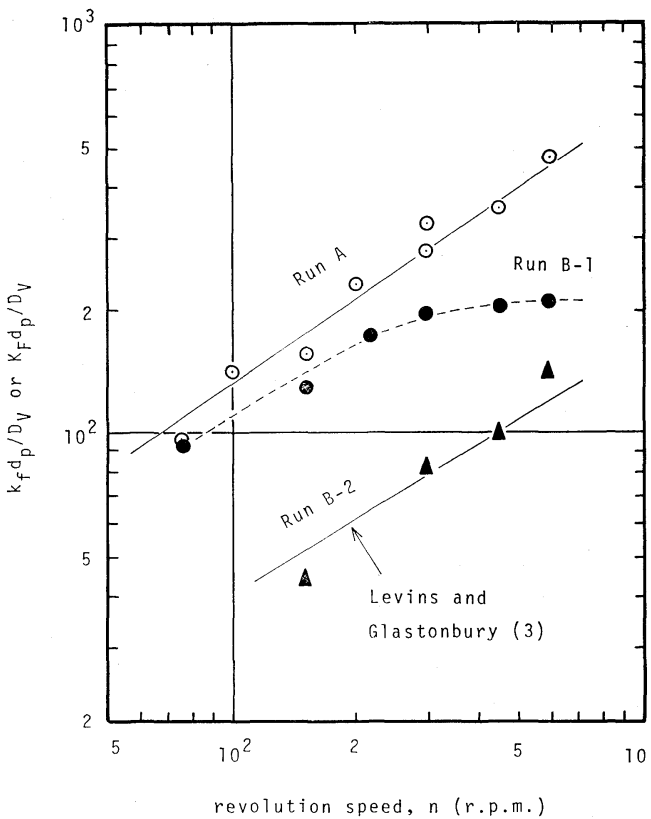

Fig. 2 Mass transfer coefficient for Run $A$ and apparent mass transfer coefficient for Runs B-1 and B-2

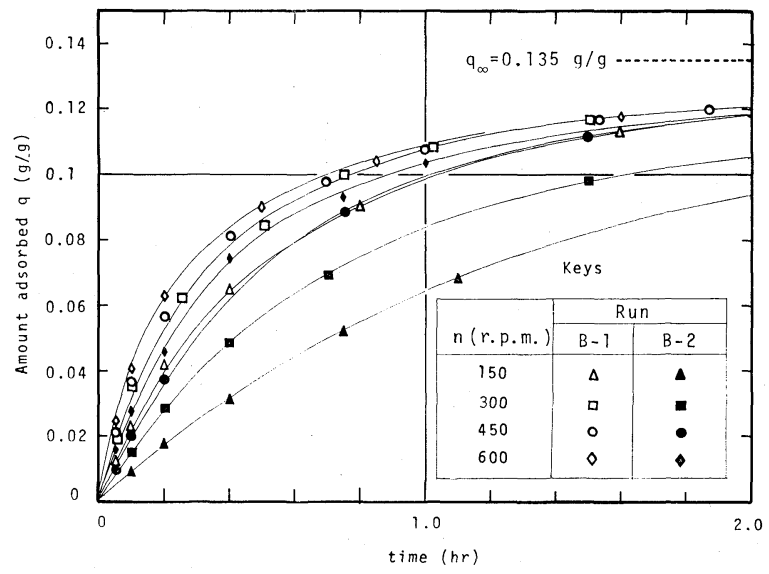

Fig. 3 Adsorption uptake of phenol by activated carbon (Runs B-1 and B-2)
A schematic illustration of the reactor is shown in Fig. 1. A small basket made of stainless steel wire mesh replaces the conventional impeller. Since the bulk volume of particles used for each run was less than $1 \mathrm{~cm}^{3}$, the size of the basket was determined as shown in Fig. 1. Dissolution of $\beta$-naphthol pellets in pure water and adsorption of phenol from an aqueous solution by activated carbon particles are employed, respectively, to determine the mass transfer coefficient from the surface of the particles in the basket to bulk liquid and to compare the behavior of the basket-type contactor and a conventional stirred tank with suspended particles.

\section{Experiment (A)}

$\beta$-naphthol pellets were prepared in a similar manner as shown by $\mathrm{Wakao}^{8}$. About 0.3 grams of cylindrical pellets ( $2.7 \mathrm{~mm}$ diameter, $4 \sim 5 \mathrm{~mm}$ length) were put into a basket made of 8-mesh stainless steel screen and concentration-time relation was measured at $75,100,150,200,300,450$ and $600 \mathrm{rpm}$ for the first 30 minutes of each run. Particle-to-liquid mass transfer resistance should be the only rate-determining step, and then the mass transfer coefficient $k_{f}$ can be calculated by the equation

$$
\frac{\left(C-C_{\text {eq. }}\right)}{\left(C_{0}-C_{\text {eq. }}\right)}=\exp \left(-\frac{k_{f} S}{V_{L}} t\right)
$$

where $S$ is the particle surface area and $V_{L}$ is the liquid volume. $C_{0}$ and $C_{\text {eq. }}$ are the initial and equilibrium concenration, respectively. The plots in Fig. 2 (hollow circles) show the thus-determined $k_{f}$ in the form of $k_{f} d_{p} / D_{V}$ versus $n$, revolution speed of the basket (rpm). Diameter of the cylindrical pellets was taken as $d_{p}$.

\section{Experiment (B)}

Initial rate of adsorption of phenol from $100 \mathrm{ppm}$ aqueous solution by activated carbon particles, CAL \#14/20 (average particle diameter $1.02 \mathrm{~mm}$ ) was measured. A basket-type contactor similar to the one used in Experiment (A), except that the basket was made of \#30 screen, was used for Run B-1 series.

A two-blade solid stainless steel impeller of the same width and height replaced the basket for Run B-2 series, and carbon particles of the same size and amount as in Run B-1 were suspended in the tank. The amount of carbon used was fixed at $0.4 \mathrm{~g}$ for each series of run.

Typical plots of the amount adsorbed, $q$, calculated from concentration change against time $t$, are shown in Fig. 3. For Runs B-1 and B-2, it is clear from the plot that the basket-type contactor gives improved performance regarding the rate in the course of adsorption. For the concentration decay or adsorp- 
tion uptake in these systems strict analysis of the whole range of experimental time is rather complicated and needs sophisticated treatment ${ }^{5)}$. However, since the purpose of this experiment is to compare a basket-type agitator with a suspension system and only the external mass transfer is of interest, no detailed discussion of intraparticle diffusion is made here. For the concentration decay or adsorption uptake, the differential form of Eq. (1) was applied to determine the apparent mass transfer coefficient, $K_{F}$ at time $t=0$. Since $C_{\mathrm{eq}}$ at the initial stage of adsorption can be taken to be zero, the following equation is used to determine $K_{F}$ from the intial concentration decay.

$$
\left[\frac{\partial \ln \left(C / C_{0}\right)}{\partial t}\right]_{t=0}=-\frac{K_{F} S}{V_{L}}
$$

Thus-determined $K_{F}$ values are included in Fig. 2 in a similar way as was done for Run A. The highest possible value of $K_{F}$ should be defined by the initial rate of intraparticle diffusion of phenol into activated carbon particles. This rate is supposed to be independent of revolution speed, $n$. For the suspended system (Run B-2), the upper limit of $K_{F}$ was determined by the possible upper limit of revolution speed of the impeller since at high speed (about $600 \mathrm{rpm}$ ) cavitation in a tank occurs together with particle attrition due to mechanical impact of the particles on the blades or the baffles. In the case of B-2-600 rpm run, $30 \mathrm{wt} \%$ of the particles passed through \#14 screen, after 90 minutes' operation. All the runs including Run A were made in a $30^{\circ} \mathrm{C}$ constant-temperature bath. The energy input for the three systems used here was measured by a simplified torque meter (Yamazaki Co.). The results are shown in Fig. 4.

\section{Discussion}

From Run A (dissolution of $\beta$-naphthol), and Run B- 1 it is estimated that particle-to-liquid mass transfer coefficient in a basket impeller is proportional to $n^{2 / 3}$,

$$
\frac{k_{f} d_{p}}{D_{V}} \propto n^{2 / 3}
$$

Since the basket-type impeller effectively utilizes the region of high-energy dissipation in a stirred $\operatorname{tank}^{2)}$ for particle surface to bulk liquid mass transfer, it must accomplish the highest rate of mass transfer possible for a given energy input. In other words, by using a basket impeller the same mass transfer rate is possible with far smaller energy input than by a conventional suspended system. At a low speed of revolution, the implicit assumption of complete mixing in a bulk liquid may become unreasonable.
Fig. 4 Power input to the reactors employed in Runs A, B-1 and B-2

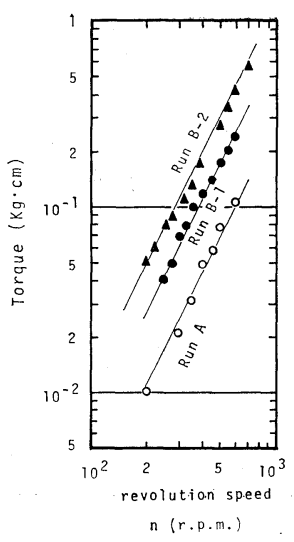

However, this assumption is justified by the calculation of mixing time in the tank. At the lowest speed (75 rpm) for Run A, the life of the biggest possible eddy in the tank, whose size is $\lambda \approx 10 \mathrm{~cm}$, is roughly estimated to $b^{6)}$

$$
\theta=\frac{3}{2}\left(\frac{\lambda^{2}}{\varepsilon}\right)^{2 / 3}=3.5 \mathrm{sec}
$$

which is far smaller than the characteristic time of the external mass transfer, since $V_{L} / k_{f} S$ for all the runs made here was greater than $15 \mathrm{~min}$. For the suspended system (Run B-2), the mass transfer cofficients are compared with the correlation proposed by Levins and Glastonbury ${ }^{4}$ in Fig. 2. The agreement is excellent. Deviation of the data for $150 \mathrm{rpm}$ can be attributed to non-homogeneous suspension of the particles due to insufficient agitation.

From Figs. 2-4 it is concluded that a basket impeller offers a better way to attain good contact between bulk fluid and particle surface than does a conventional suspension system. Also, this type of contactor may be effective for many other applications, such as operation of mass transfer processes involving solid particles in a large volume of liquid, while a suspension system can be used without significant disadvantage when intraparticle diffusion rates are slow $^{5}$.

\section{Acknowledgment}

Mr. Toshio Miyazaki, Institute of Industrial Science, University of Tokyo, was helpful in doing the experiment.

\section{Literature Cited}

1) Bennett, C. O., M. B. Cutlip and C. C. Yang: Chem. Eng. Sci., 27, 2255 (1972).

2) Cutter, L. A.: AIChE J., 12, 35 (1966).

3) Furusawa, T. and J. M. Smith: Ind. Eng. Chem., Fundamentals, 12, 197 (1973).

4) Levins, D. M. and J. R. Glastonbury: Trans. Inst. Chem. Engrs., 50, 132 (1972).

5) Suzuki, M. and K. Kawazoe: J. Chem. Eng. Japan, 7, 346.

6) Suzuki, M.: J. Chem. Eng. Japan, 4, 354 (1971).

7) Tajbl, D. G., J. B. Simmons and J. J. Carberry: Ind. Eng. Chem. Fundamentals, 5, 171 (1966). 